\title{
Definition of Complexity Factor for Self-Gravitating Systems in Palatini $f(R)$ Gravity
}

\author{
Z. Yousaf* \\ Department of Mathematics, University of the Punjab, \\ Quaid-i-Azam Campus, Lahore-54590, Pakistan.
}

\begin{abstract}
The aim of this paper is to explore the complexity factor for those self-gravitating relativistic spheres whose evolution proceeds non- dynamically. We are adopting the definition of CF mentioned in [50], modifying it to the static spherically symmetric case, within the framework of a modified gravity theory (the Palatini $f(R)$ theory). In this respect, we have considered radial dependent anisotropic matter content coupled with spherical geometry and determined the complexity factor involved in the patterns of radial evolution. We shall explore the field and a well-known Tolman-Oppenheimer-Volkoff equations. After introducing structure scalars from the orthogonal decomposition of the Riemann tensor, we shall calculate complexity factor. An exact analytical model is presented by considering firstly ansatz provided by Gokhroo and Mehra. The role of matter variables and $f(R)$ terms are analyzed in the structure formation as well as their evolution through a complexity factor.
\end{abstract}

Keywords: Self-gravitating system; Anisotropy; Complexity factor. PACS: 04.40.-b; 04.40.Dg; 04.50.Kd

*zeeshan.math@pu.edu.pk 


\section{Introduction}

One of the most important gravitational theories, presented by Albert Einstein about more than a century ago is general relativity (GR). This theory has rejected Newton's idea of gravitational force and described the deformation of space-time geometry through the mass-energy distribution. The recent plethora of observations, like, type Ia supernovae, large scale structure and cosmic microwave background radiation [1 3], states that our cosmos is characterized by an accelerated expansion. Furthermore, various recent cosmic observations at astronomical scales [4,5] confirmed the unknown nature of the most matter part of the universe.

After the observational works presented by LIGO/Virgo collaboration for understanding neutron star mergers [6], binary compact systems [7, 8], and the initiative probes of EUCLID [9], the cosmic models provided by modified theories of gravity (MTG) will be put to experimental verification in extragalactic and cosmological backgrounds. Many cosmological models of MGT have been ruled out by current observational outcomes from the mergers of neutron stars. Not only this, the severe restriction has also been imposed by such experiments on the viability of some MTG [10,11. There has been a number of achievements of GR, however, the search for detection of dark matter/energy sources in view of the concordance model, space-time singularities [12], etc may require some MTG. Keeping in mind the presence of unobserved (up to this time) dark matter (DM) and dark energy (DE), a new route of research was suggested based on the generalizations of GR. This famous idea has received standard terminology called MTG.

Qadir et al. [13] also suggested that GR may need to extend in order to study the various aspects of gravitation with quantum cosmology. There has been very interesting cosmological models related to MTG for the description of stellar collapse and their evolution [14-18. Nojiri and Odintsov [19] described not only the significance of MGT but also elaborated the applications of such theories in the discussion of comic evolution. The straightforward MTG from GR is $f(R)$ ( $R$ is the Ricci scalar) [15, 20, and $f(\mathcal{T})(\mathcal{T}$ is the torsion scalar) [21]. After which, such theories are generalized by including amalgams of curvature terms in a more complex ways, for instance, $f(R, \square R, T)$ ( $\square$ is the de Alembert's operator and $T$ is the trace of energy momentum tensor) [22, 23], $f(G)$ ( $G$ is the Gauss-Bonnet term) [24] and $f(G, T)$ [25-28] etc. (details of MTG can be seen in, 29 33]). The considerable amount of research about the introduction as well the application of 
Palatini $f(R)$ scheme is available in literature [34 37].

Gravitational collapse is one of the interesting phenomena of the stellar systems in which the matter of the massive structure moves towards its center as a consequence of a force produced by its own gravitational pull. Stars, star clusters and galaxies could be the final fates of this process from the interstellar gas. The inhomogeneous state of energy density is believed to be the progenitors of the stellar collapse. Thus, the importance of the study of inhomogeneous energy density in the study of collapse is justified. Penrose [38] was curious to understand radiating self-gravitating systems from the clumped matter distribution. He expressed the heterogeneous energy density through Weyl curvature tensor and described gravitation in terms of entropy.

Eardley and Smarr [39] calculated exact solution of a non-static spherical dust cloud in order to explore the importance of the irregular distribution of energy density and claimed that such a relativistic collapse is likely to end up with a new type of naked singularity. Herrera et al. [40] used an analytical approach to evaluate the expression of active gravitational mass supporting the existence of heterogeneous energy density for the relativistic radiating spheres. Herrera et al. [41] orthogonal splitting of Riemann tensor and found few scalars associated supporting the existence of heterogeneous energy density for the relativistic radiating spheres. Bamba et al. 42] calculated the rate of spherical collapse in MTG and discussed some various characteristics of curvature singularity during its collapse. Yousaf et al. studied the role of tilted congruences [43,44] as well as MTG on the existence of regular energy density [45,46], and pace of gravitational collapse [47 49]. They inferred that extra curvature terms due to MTG tend to slow the collapse process.

In the continuation of the study of energy density irregularities, Herrera [50] introduced a new concept for the study of homogeneous distribution of static systems with the help of a factor. He called this factor as a complexity factor $(\mathrm{CF})$ and expressed it through the structure scalars. Abbas and Nazar [51] applied the same procedure in order to present the definition CF for the anisotropic system in a particular MTG gravity. Then, Herrera et al. [52] generalized their concept for dynamical self-gravitating systems. Recently, Sharif and Majid [53] and Yousaf et al. [54,55] modified their results in the Brans-Dicke theory and $f\left(R, T, R_{\mu \nu} T^{\mu \nu}\right)$ gravity, respectively and analyzed the role of modified terms in the formulation of $\mathrm{CF}$.

We are adopting the definition of CF mentioned in [50], generalizing it to the static spherically symmetric case, within the context of a modified gravity theory (the Palatini $f(R)$ theory). This work is devoted to under- 
standing the role of Palatini $f(R)$ terms in the theoretical modeling of static self-gravitating systems. In this regard, we shall present $\mathrm{CF}$ and then this function in terms of structure scalars. The paper is outlined below. In the coming section, we shall compute Palatini $f(R)$ equations of motion and hydrostatic equation. After calculating the Misner-Sharp mass function in the same section, we shall express the Tolman mass through usual and effective matter variables. Section 3 is devoted to the orthogonal splitting of the curvature tensor. The scalars obtained from this calculation will then be used to define CF. Finally, we present concluding remarks.

\section{Palatini $f(R)$ Gravity and its Related Vari- ables}

The action function for the evaluation of field equation in $f(R)$ gravity is

$$
S_{f(R)}=\frac{1}{2 \kappa} \int d^{4} x \sqrt{-g} f(\hat{R})+S_{M},
$$

in which $S_{M}$ describes matter action, while $\kappa$ is a coupling constant. It is the worthy to stress that in the above action the scalar function $\hat{R}$ is assembled from the contraction of the corresponding metric tensor with that of Ricci tensor associated with the connection symbol $\left(\hat{R}:=g^{\gamma \delta} R_{\gamma \delta}\right)$, thereby indicating $\hat{R}$ through geometrical connections. The details on this account can be found from [56]. By preserving the relation $\Gamma_{\gamma \delta}^{\mu} \neq \Gamma_{\delta \gamma}^{\mu}$, the variations of Eq.(11) with $g_{\gamma \delta}$ and $\Gamma_{\gamma \delta}^{\mu}$, respectively give

$$
\begin{aligned}
& f_{R}(\hat{R}) \hat{R}_{\gamma \delta}-\left[g_{\gamma \delta} f(\hat{R})\right] / 2=\kappa T_{\gamma \delta}, \\
& \hat{\nabla}_{\mu}\left(g^{\gamma \delta} \sqrt{-g} f_{R}(\hat{R})\right)=0
\end{aligned}
$$

where $T_{\gamma \delta}$ stands for energy-momentum tensor that here does not depend on geometric connections. Its value can be found as

$$
T_{\gamma \delta}=-2(-g)^{-1 / 2} \frac{\delta S_{M}}{\delta g^{\gamma \delta}} .
$$

The aim of the paper is to calculate CF involved in the emergence of irregularities over the anisotropic matter distribution in Palatini $f(R)$ gravity. Therefore, we assume energy momentum in mixed form $\left(T_{\nu}^{\mu}\right)$ as

$$
T_{\nu}^{\mu(m)}=\rho u^{\mu} u_{\nu}+\Pi_{\nu}^{\mu}-P h_{\nu}^{\mu},
$$


where $u_{\gamma}$ is the fluid's four velocity and $h_{\nu}^{\mu}$ is the projection tensor. Further, $P$ is expressed as the combination of radial $P_{r}$ and tangential $P_{\perp}$ pressure components as $P=\frac{1}{3}\left(P_{r}+2 P_{\perp}\right)$, while the $\Pi_{\nu}^{\mu}$ is the anisotropic tensor which can be written through anisotropic scalar $\Pi=-\left(P_{\perp}-P_{r}\right)$ and four vector $\chi^{\mu}$ as $\Pi_{\nu}^{\mu}=\Pi\left(\chi^{\mu} \chi_{\nu}+\frac{1}{3} h_{\nu}^{\mu}\right)$.

The application of $d f / d \hat{R}$ has been described through the subscript $R$ on the associated mathematical quantities. The dependence of the Ricci invariant on $T$ with $T:=g^{\gamma \delta} T_{\gamma \delta}$ can be obtained from Eq.(2) as

$$
\hat{R} f_{R}(\hat{R})-2 f(\hat{R})=\kappa T .
$$

In the background of vacuum space, $R$ will have a constant value that can be described through $f(\hat{R})$. This makes us to define another form of metric tensor $h_{\gamma \delta}$ as $h_{\gamma \delta}:=f_{R} g_{\gamma \delta}$. This scenario describes the gravitational interaction in the $\Lambda$ dominated epoch. In this way, the cosmic features of vacuum space from $f(R)$ theory of gravity can be viewed. Thus, the geometric connection associated with $h_{\gamma \delta}$ becomes connection of Levi-Civita given by

$$
\Gamma_{\gamma \delta}^{\nu}=\frac{1}{2} h^{\nu \alpha}\left(\partial_{\gamma} h_{\alpha \delta}+\partial_{\delta} h_{\alpha \gamma}-\partial_{\alpha} h_{\gamma \delta}\right) .
$$

There is a conformal relation between tensors $h_{\gamma \delta}$ and $g_{\gamma \delta}$. In order to proceed our analysis with the second order metric equations, we compute connection from Eq.(2), which after using in Eq.(3) provides

$$
\begin{aligned}
& \frac{1}{f_{R}}\left(\hat{\nabla}_{\gamma} \hat{\nabla}_{\delta}-g_{\gamma \delta} \hat{\square}\right) f_{R}+\frac{1}{2} g_{\gamma \delta} \hat{R}+\frac{\kappa}{f_{R}} T_{\gamma \delta}+\frac{1}{2} g_{\gamma \delta}\left(\frac{f}{f_{R}}-\hat{R}\right) \\
& +\frac{3}{2 f_{R}^{2}}\left[\frac{1}{2} g_{\gamma \delta}\left(\hat{\nabla} f_{R}\right)^{2}-\hat{\nabla}_{\gamma} f_{R} \hat{\nabla}_{\delta} f_{R}\right]-\hat{R}_{\gamma \delta}=0,
\end{aligned}
$$

while $\hat{\nabla}_{\gamma}$ indicates covariant derivation with respect to $g_{\gamma \delta}$, $\hat{\square}_{\text {is the d'Alembertian }}$ and $f_{\mathcal{P}}=\frac{d f(\mathcal{P})}{d \mathcal{P}}$. Equation (8) is a single set of field equation that can be interpreted through Einstein tensor $\left(G_{\gamma \delta}\right)$ as

$$
\hat{G}_{\gamma \delta}=\frac{\kappa}{f_{R}}\left(T_{\gamma \delta}+\mathcal{T}_{\gamma \delta}\right)
$$

where

$$
\mathcal{T}_{\gamma \delta}=\frac{1}{\kappa}\left(\hat{\nabla}_{\gamma} \hat{\nabla}_{\delta}-g_{\gamma \delta} \hat{\square}\right) f_{R}-\frac{f_{R}}{2 \kappa} g_{\gamma \delta}\left(\hat{R}-\frac{f}{f_{R}}\right)
$$




$$
+\frac{3}{2 \kappa f_{R}}\left[\frac{1}{2} g_{\gamma \delta}\left(\hat{\nabla} f_{R}\right)^{2}-\hat{\nabla}_{\gamma} f_{R} \hat{\nabla}_{\delta} f_{R}\right] .
$$

The system under consideration consists of a static spherically symmetric spacetime whose line element can be given as follows

$$
d s^{2}=e^{v} d t^{2}-e^{\omega} d r^{2}-r^{2}\left(d \theta^{2}+\sin ^{2} \theta d \phi^{2}\right),
$$

where $v$ and $\omega$ are functions of radial coordinate only. The derivation with respect to $r$ will be described through prime in this paper. Under comoving reference system, we define four vectors through metric coefficients as

$$
\chi^{\mu}=\left(0, e^{\frac{-\omega}{2}}, 0,0\right)
$$

obeying

$$
\chi^{\gamma} \chi_{\gamma}=-1, \quad \chi^{\gamma} u_{\gamma}=0 .
$$

The non-zero $f(R)$ equations of motion (9) for the metric (10) are

$$
\begin{aligned}
& \frac{1}{f_{R}}\left[8 \pi \mu+\left(\frac{f-R f_{R}}{2}\right)-\frac{3 f_{R}^{\prime 2}}{4 f_{R} e^{\omega}}-\frac{v^{\prime} f_{R}^{\prime}}{2 e^{\omega}}-\frac{f_{R}^{\prime 2}}{e^{\omega}}+\frac{f_{R}^{\prime \prime}}{e^{\omega}}\right] \\
& =\frac{1}{r^{2}}+e^{-\omega}\left(\frac{\omega^{\prime}}{r}-\frac{1}{r^{2}}\right), \\
& \frac{1}{f_{R}}\left[8 \pi P_{r}+\left(\frac{f-R f_{R}}{2}\right)+\frac{5 f_{R}^{\prime 2}}{4 f_{R} e^{\omega}}+\frac{f_{R}^{\prime} \omega^{\prime}}{2 e^{\omega}}\right] \\
& =\frac{1}{r^{2}}+e^{-\omega}\left(\frac{v^{\prime}}{r}+\frac{1}{r^{2}}\right), \\
& \frac{1}{f_{R}}\left[32 \pi P_{\perp}+4\left(\frac{f-R f_{R}}{2}\right)-\frac{3 f_{R}^{\prime}}{4 f_{R} e^{\omega}}-\frac{f_{R}^{\prime}}{r e^{\omega}}-\frac{f_{R}^{\prime}}{e^{\omega}}+\frac{f_{R}^{\prime \prime}}{e^{\omega}}\right] \\
& =-e^{-\omega}\left\{\omega^{\prime} v^{\prime}-2 v^{\prime \prime}+\frac{2 \omega^{\prime}}{r}-v^{\prime 2}-\frac{2 v^{\prime}}{r}\right\} .
\end{aligned}
$$

Here we take $\kappa=8 \pi$.

\section{$3 \quad$ Hydrostatic Equation and Mass Functions}

In this section, we shall compute mass functions described by Misner-Sharp [57] and Tolman [58]. Later on, we express these functions in terms of matter 
and dark source variables of the spherically symmetric spacetime. One can calculate the conservation laws from Bianchi identities with respect to usual and effective stress-energy tensors as

$$
P_{r}^{\prime}=\frac{2}{r}\left(P_{\perp}-P_{r}\right)-\frac{\beta^{\prime}}{2}\left(\rho+P_{r}-T_{1}^{1(\mathcal{P})}+T_{0}^{0(\mathcal{P})}\right)+\frac{2}{r}\left(T_{1}^{1(\mathcal{P})}-T_{2}^{2(\mathcal{P})}\right)+D_{0},
$$

where

$$
D_{0}=\left(-\frac{f_{R}^{\prime}}{2 f_{R}}-\frac{\omega^{\prime}}{2}\right)\left(T_{1}^{1(\mathcal{P})}-T_{0}^{0(\mathcal{P})}\right)+\left(\frac{2}{r}+\frac{f_{R}^{\prime}}{f_{R}}\right)\left(T_{1}^{1(\mathcal{P})}-T_{2}^{2(\mathcal{P})}\right)+\left(T_{1}^{1(\mathcal{P})}\right)_{, 1} .
$$

The total quantity of relativistic matter content for the spherical geometry can be calculated through the formalism provided by Misner-Sharp as [57]

$$
m(r)=\frac{r}{2}\left(1-e^{-\omega}\right),
$$

which can be reexpressed after making use of Eq.(13) as

$$
m(r)=4 \pi \int_{0}^{r} \frac{r^{2}}{f_{R}}\left(\rho+T_{0}^{0(\mathcal{P})}\right) d r .
$$

Using Eqs.(13)-(15) and Eq.(18), it follows that

$$
\begin{aligned}
m & =\frac{4 \pi}{3 f_{R}} r^{3}\left(\rho-P_{r}-T_{1}^{1(\mathcal{P})}+P_{\perp}+T_{0}^{0(\mathcal{P})}+T_{2}^{2(\mathcal{P})}\right) \\
& -\frac{r^{3}}{3}\left[\frac{1}{4} e^{-\omega}\left(v^{\prime \prime}+\frac{v^{\prime 2}}{2}+\frac{\omega^{\prime}}{r}+\frac{2}{r^{2}}-\frac{\omega^{\prime} v^{\prime}}{2}-\frac{v^{\prime}}{r}-\frac{2 e^{\omega}}{r^{2}}\right)\right] .
\end{aligned}
$$

The well-known Weyl curvature tensor can be written through its electric part $\left(E^{\sigma \chi}\right)$ and Levi-Civita tensor $\left(\eta_{\mu \nu \gamma \sigma}\right)$ as

$$
C_{\mu \nu \xi \lambda}=\left(g_{\mu \nu \gamma \sigma} g_{\xi \lambda \tau \chi}-\eta_{\mu \nu \gamma \sigma} \eta_{\xi \lambda \tau \chi}\right) u^{\gamma} u^{\tau} E^{\sigma \chi}, \quad \gamma, \sigma, \tau,
$$

where $g_{\mu \nu \gamma \sigma}:=g_{\mu \gamma} g_{\nu \sigma}-g_{\mu \sigma} g_{\nu \gamma}$. One can write $E^{\sigma \chi}$ in another way as

$$
E_{\mu \nu}=C_{\mu \xi \nu \lambda} u^{\xi} u^{\lambda},
$$

whose value with the help of Weyl scalar turns out to be

$$
E^{\sigma \chi}=\mathcal{E}\left(\frac{1}{3} h^{\sigma \chi}+\chi^{\sigma} \chi^{\chi}\right)
$$


Its value through structural variables is found as follows

$$
\mathcal{E}=-\frac{1}{4} e^{-\omega}\left[v^{\prime \prime}+\frac{v^{\prime 2}}{2}+\frac{\omega^{\prime}}{r}+\frac{2}{r^{2}}-\frac{\omega^{\prime} v^{\prime}}{2}-\frac{v^{\prime}}{r}-\frac{2 e^{\omega}}{r^{2}}\right]
$$

The expression mentioning in Eq.(18) can be manipulated through this scalar as

$$
m=\frac{4 \pi}{3 f_{R}} r^{3}\left(\rho-P_{r}-T_{1}^{1(\mathcal{P})}+P_{\perp}+T_{0}^{0(\mathcal{P})}+T_{2}^{2(\mathcal{P})}\right)+\frac{1}{3} r^{3} \mathcal{E},
$$

from which $\mathcal{E}$ becomes

$\mathcal{E}=\frac{4 \pi}{f_{R}}\left(P_{r}-T_{2}^{2(\mathcal{P})}-P_{\perp}+T_{1}^{1(\mathcal{P})}\right)-\frac{4 \pi}{r^{3}} \int_{0}^{r} r^{3}\left(\frac{\rho}{f_{R}}\right)^{\prime} d r-\frac{4 \pi}{r^{3}} \int_{0}^{r} r^{3}\left(\frac{T_{0}^{0(\mathcal{P})}}{f_{R}}\right)^{\prime} d r$

This expression has been expressed through matter variables and Palatini $f(R)$ corrections. Such a relation could be helpful to analyze the heterogenous state of energy density. This equation could provide information about the existence of regular energy density during the subsequent evolution of the spherical static cloud. Equation (25) along with the above equation gives

$$
m=\frac{4 \pi r^{3}}{3 \tilde{f}_{R}}\left(\rho+T_{0}^{0(\mathcal{P})}\right)-\frac{4 \pi}{3 \tilde{f}_{R}} \int_{0}^{r} r^{3}\left(\rho+T_{0}^{0(\mathcal{P})}\right)^{\prime} d r
$$

where tilde states that the quantity is being evaluated at the present Ricci scalar condition. This relation has directly related mass function, effective form of regular spherical energy density and the role of heterogeneous energy density on the stability of relativistic spherical interiors.

The value of one of the metric coefficients found in Eq.(10) can be calculated from the field equation and Eq.(18) as

$$
\beta^{\prime}=2 \frac{m f_{R}+4 \pi r^{3}\left(P_{r}+T_{1}^{1(\mathcal{P})}\right)}{r(r-2 m)} .
$$

We now use Eq.(28) in Eq.(16) to obtain Tolman-Opphenheimer-Volkoff (TOV) equation in the presence of Palatini $f(R)$ corrections as

$$
P_{r}^{\prime}=T_{1,1}^{1(\mathcal{P})}-\frac{m f_{R}+4 \pi r^{3}\left(P_{r}+T_{1}^{1(\mathcal{P})}\right)}{r(r-2 m)}\left(\rho+P_{r}+T_{0}^{0(\mathcal{P})}-T_{1}^{1(\mathcal{P})}\right)
$$




$$
+\frac{2}{r}\left(P_{\perp}-P_{r}+T_{1}^{1(\mathcal{P})}-T_{2}^{2(\mathcal{P})}\right) .
$$

We now suppose that our system is comprised of boundary surface at $r=r_{\Omega}$, denoted by $\Omega$, which has demarcated our manifolds into two parts, the exterior and the interior one. The exterior region can be described through the following spacetime

$$
d s^{2}=\left(1-\frac{2 \mathcal{M}}{r}\right) d t^{2}-\frac{d r^{2}}{\left(1-\frac{2 \mathcal{M}}{r}\right)}-r^{2}\left(d \theta^{2}+\sin ^{2} \theta d \phi^{2}\right),
$$

while the interior to $\Omega$ is described by Eq.(10). In Eq.(30), $\mathcal{M}$ describes the mass of the gravitating source. The fundamental forms of Darmois junction conditions [59] at $r=r_{\Omega}$ provides the smooth matching of (10) and (30) manifolds. These give

$$
e^{v_{\Omega}}=1-\frac{2 \mathcal{M}}{r_{\Omega}}, \quad e^{\omega_{\Omega}}=\left(1-\frac{2 \mathcal{M}}{r_{\Omega}}\right)^{-1}, \quad\left[P_{r}\right]_{\Omega}=0 .
$$

Herrera and Santos [60] described the formula provided by Tolman [58] in order to analyze the amount of matter content within the spherical geometric distribution. Further, Herrera et al. [40] expressed this relation in terms of structural properties of self-gravitating system. They also checked its role in the maintenance of homogeneous energy density over the boundary. One can write Tolman formula with Palatini $f(R)$ terms as

$$
m_{T}=4 \pi \int_{0}^{r_{\Omega}} \frac{r^{2}}{f_{R}} e^{\frac{v+\omega}{2}}\left(\rho+P_{r}+2 P_{\perp}+T_{0}^{0(\mathcal{P})}+T_{1}^{1(\mathcal{P})}+2 T_{2}^{2(\mathcal{P})}\right) d r .
$$

For the bounded system, it follows from the above equation that

$$
m_{T}=4 \pi \int_{0}^{r} \frac{r^{2}}{f_{R}} e^{\frac{v+\omega}{2}}\left(\rho+P_{r}+2 P_{\perp}+T_{0}^{0(\mathcal{P})}+T_{1}^{1(\mathcal{P})}+2 T_{2}^{2(\mathcal{P})}\right) .
$$

It could be regarded as the corresponding active gravitational mass. By making use of Eqs.(13)-(15) in Eq.(32), we have

$$
m_{T}=\frac{r^{2}}{2 \tilde{f}_{R}} e^{\frac{v-\omega}{2}} v^{\prime}-\frac{4 \pi}{\tilde{f}_{R}} \int_{0}^{r} e^{\frac{v+\omega}{2}} r^{2}\left(2 T_{2}^{2(\mathcal{P})}+T_{1}^{1(\mathcal{G})}-T_{0}^{0(\mathcal{P})}\right) d r .
$$

Using Eq.(28) in Eq.(33), we get

$$
m_{T}=\left(4 \pi r^{3}\left(P_{r}+T_{1}^{1(\mathcal{P})}\right)+m \tilde{f}_{R}\right) \frac{e^{\frac{v+\omega}{2}}}{\tilde{f}_{R}}
$$




$$
-\frac{4 \pi}{\tilde{f}_{R}} \int_{0}^{r} e^{\frac{v+\omega}{2}} r^{2}\left(2 T_{2}^{2(\mathcal{P})}+T_{1}^{1(\mathcal{P})}-T_{0}^{0(\mathcal{P})}\right) d r
$$

Then the corresponding $m_{T}$ for the anisotropic system turns out to be

$$
\begin{aligned}
m_{T} & =\left(\frac{r}{r_{\Omega}}\right)^{3}\left[m_{T}\right]_{\Omega}+r^{3} \int_{r}^{r_{\Omega}} \frac{e^{\frac{v+\omega}{2}}}{r}\left[\frac{4 \pi}{\tilde{f}_{R}}\left(P_{\perp}-P_{r}\right)-\mathcal{E}\right] d r \\
& +\frac{4 \pi}{\tilde{f}_{R}} r^{3} \int_{r}^{r_{\Omega}} e^{\frac{v+\omega}{2}}\left(T_{0}^{0(\mathcal{P})}+4 T_{1}^{1(\mathcal{P})}+2 T_{2}^{2(\mathcal{P})}\right) d r \\
& -r^{3} \frac{4 \pi}{\tilde{f}_{R}} \int_{0}^{r} e^{\frac{v+\omega}{2}} r^{2}\left(T_{0}^{0(\mathcal{P})}-T_{1}^{1(\mathcal{P})}-2 T_{2}^{2(\mathcal{P})}\right) d r .
\end{aligned}
$$

This equation states that the value of Tolman mass depends mainly on the participation of fluid energy density, $f(R)$ dark source terms and pressure anisotropy of the spherical relativistic geometric distribution.

\section{Structure Scalars}

The definition for Riemann tensor can be written as follows

$R_{\xi \nu \lambda}^{\mu}=C_{\xi \nu \lambda}^{\mu}+\frac{1}{2} R_{\nu}^{\mu} g_{\xi \lambda}-\frac{1}{2} R_{\xi \nu} \delta_{\lambda}^{\mu}+\frac{1}{2} R_{\xi \lambda} \delta_{\nu}^{\mu}-\frac{1}{2} R_{\lambda}^{\mu} g_{\xi \nu}-\frac{1}{6} R\left(\delta_{\nu}^{\mu} g_{\xi \lambda}-\delta_{\lambda}^{\mu} g_{\xi \nu}\right)$,

where $R, C_{\mu \xi \nu \lambda}$ and $R_{\mu \nu}$ stand for the Ricci scalar, the Weyl tensor and the Ricci tensor. One can manipulate Eq.(36) as

$$
R_{\nu \delta}^{\mu \gamma}=C_{\nu \delta}^{\mu \gamma}+2 T_{[\nu}^{(t o t)[\mu} \delta_{\delta]}^{\gamma]}+T^{(t o t)}\left(\frac{1}{3} \delta_{[\nu}^{\mu} \delta_{\delta]}^{\gamma}-\delta_{[\nu}^{[\mu} \delta_{\delta]}^{\gamma]}\right) .
$$

Herrera et al. [61] calculated some scalar variables from the Riemann tensor splitting. This technique has been proved to be very helpful in understanding the basic ingredients of matter content of the self-gravitating system. For this purpose, we took few tensorial quantities as follows [61]

$$
\begin{aligned}
Y_{\mu \nu} & =R_{\mu \xi \nu \lambda} u^{\xi} u^{\lambda}, \\
X_{\mu \nu} & ={ }^{*} R_{\mu \xi \nu \lambda}^{*} u^{\xi} u^{\lambda}=\frac{1}{2} \eta_{\mu \nu}^{\alpha \beta} R_{\alpha \beta \xi \lambda}^{*} u^{\xi} u^{\lambda},
\end{aligned}
$$

where $R_{\mu \nu \xi \lambda}^{*}=\frac{1}{2} \eta_{\alpha \beta \xi \lambda} R_{\mu \nu}^{\alpha \beta}$. Equation (37) can be written alternatively as follows

$$
R_{\nu \lambda}^{\mu \xi}=R_{(I) \nu \lambda}^{\mu \xi}+R_{(I I) \nu \lambda}^{\mu \xi}+R_{(I I I) \nu \lambda}^{\mu \xi}+R_{(I V) \nu \lambda}^{\mu \xi}+R_{(V) \nu \lambda}^{\mu \xi},
$$


where

$$
\begin{aligned}
R_{(I) \nu \lambda}^{\mu \xi} & =\frac{16 \pi}{\tilde{f}_{R}} \rho u^{[\mu} u_{[\nu} \delta_{\lambda]}^{\xi]}+\frac{8 \pi}{\tilde{f}_{R}}(\rho-3 P)\left(\frac{1}{3} \delta_{[\nu}^{\mu} \delta_{\lambda]}^{\xi}-\delta_{[\nu}^{[\mu} \delta_{\lambda]}^{\xi]}\right) \\
& -16 \pi P h_{[\nu}^{[\mu} \delta_{\lambda]}^{\xi]}, \\
R_{(I I) \nu \lambda}^{\mu \xi} & =\frac{16 \pi}{\tilde{f}_{R}} \Pi_{[\nu}^{[\mu} \delta_{\lambda]}^{\xi]} \\
R_{(I I I) \nu \lambda}^{\mu \xi} & =4 u^{[\mu} u_{[\nu} E_{\lambda]}^{\xi]}-\epsilon_{\alpha}^{\mu \xi} \epsilon_{\nu \lambda \beta} E^{\alpha \beta} \\
R_{(I V) \nu \lambda}^{\mu \xi} & =4\left[R_{\gamma \eta} \delta_{[\nu}^{[\mu} \delta_{\lambda]}^{\xi]}+g_{\gamma \eta} \delta_{[\nu}^{[\mu} \delta_{\lambda]}^{\xi]}-R_{\gamma \eta[\nu}^{[\mu} \delta_{\lambda]}^{\xi]}-R_{\gamma[\nu} \delta_{\eta}^{[\mu} \delta_{\lambda]}^{\xi]}-R_{\eta}^{[\mu} g_{\gamma[\nu} \delta_{\lambda]}^{\xi]}\right. \\
& \left.-\frac{1}{2} R\left(\delta_{[\nu}^{[\mu} g_{\gamma \eta} \delta_{\lambda]}^{\xi]}+\delta_{\eta}^{[\mu} g_{\gamma[\nu} \delta_{\lambda]}^{\xi]}\right)\right] \nabla^{\gamma} \nabla^{\eta} f_{R}+\left(f-R f_{R}\right) \delta_{[\nu}^{[\mu} \delta_{\lambda]}^{\xi]},(43) \\
R_{(V) \nu \lambda}^{\mu \xi} & =4\left[\left(R_{\gamma \eta}-\frac{1}{2} R g_{\gamma \eta}\right) \nabla^{\gamma} \nabla^{\eta} f_{R}+\left(f-R f_{R}\right)\right]\left(\frac{1}{3} \delta_{[\nu}^{\mu} \delta_{\lambda]}^{\xi}-\delta_{[\nu}^{[\mu} \delta_{\lambda]}^{\xi]}\right),(4)
\end{aligned}
$$

and $\epsilon_{\nu \lambda \beta}=u^{\mu} \eta_{\mu \nu \lambda \beta}$ with $\epsilon_{\nu \lambda \beta} u^{\beta}=0$. By making use of Eqs.(40)-(44) in Eqs.(38) and (39), we get

$$
\begin{aligned}
& X_{\mu \nu}^{(t o t)}=X_{\mu \nu}^{(m)}+X_{\mu \nu}^{(\mathcal{P})} \\
& Y_{\mu \nu}^{(t o t)}=Y_{\mu \nu}^{(m)}+Y_{\mu \nu}^{(\mathcal{P})}
\end{aligned}
$$

where the terms with superscripts $m$ and $\mathcal{P}$ indicates that the corresponding terms are related to usual matter and Palatini $f(R)$ terms, respectively. These may be written as

$$
\begin{aligned}
& X_{\mu \nu}^{(t o t)}=\frac{8 \pi}{3 \tilde{f}_{R}} \rho h_{\mu \nu}+\frac{4 \pi}{\tilde{f}_{R}} \Pi_{\mu \nu}-E_{\mu \nu}+X_{\mu \nu}^{(\mathcal{P})}, \\
& Y_{\mu \nu}^{(t o t)}=\frac{4 \pi}{3 \tilde{f}_{R}}(\rho+3 P) h_{\mu \nu}+\frac{4 \pi}{\tilde{f}_{R}} \Pi_{\mu \nu}+E_{\mu \nu},
\end{aligned}
$$

where $Y_{\mu \nu}^{(t o t)}=Y_{\mu \nu}^{(m)}[50]$. One can write both trace and trace-less components from the above equations as

$$
\begin{aligned}
X_{\mu \nu} & =X_{T F}\left(\frac{1}{3} h_{\mu \nu}+s_{\mu} s_{\nu}\right)+\frac{1}{3} X_{T} h_{\mu \nu} \\
Y_{\mu \nu} & =Y_{T F}\left(\frac{1}{3} h_{\mu \nu}+s_{\mu} s_{\nu}\right)+\frac{1}{3} Y_{T} h_{\mu \nu}
\end{aligned}
$$


from where $Y_{T}$ and $Y_{T F}$ after using the Palatini $f(R)$ field equations can be found as follows

$$
\begin{aligned}
Y_{T} & =\frac{4 \pi}{\tilde{f}_{R}}\left(\rho-2 \Pi+3 P_{r}\right), \\
Y_{T F} & =\frac{4 \pi}{\tilde{f}_{R}} \Pi+\mathcal{E} .
\end{aligned}
$$

Equations (26) and (52) yield

$Y_{T F}=\frac{8 \pi}{\tilde{f}_{R}} \Pi+\frac{4 \pi}{\tilde{f}_{R}}\left(T_{1}^{1(\mathcal{P})}-T_{2}^{2(\mathcal{P})}\right)-\frac{4 \pi}{r^{3}} \int_{0}^{r} r^{3}\left(\frac{\rho}{f_{R}}\right)^{\prime} d r-\frac{4 \pi}{r^{3}} \int_{0}^{r} r^{3}\left(\frac{T_{0}^{0(\mathcal{P})}}{f_{R}}\right)^{\prime} d r$.

These scalar quantities were defined and discussed in detail for the first time by Herrera et al. 61]. The expression of $m_{T}$ can be written after using Eqs. (152) and (35) as

$$
\begin{aligned}
m_{T} & =\left(\frac{r}{r_{\Omega}}\right)^{3}\left[m_{T}\right]_{\Omega}+r^{3} \int_{r}^{r_{\Omega}} \frac{e^{\frac{v+\omega}{2}}}{r} Y_{T F} d r \\
& +\frac{4 \pi}{\tilde{f}_{R}} r^{3} \int_{r}^{r_{\Omega}} e^{\frac{v+\omega}{2}}\left(T_{0}^{0(\mathcal{P})}+4 T_{1}^{1(\mathcal{P})}+2 T_{2}^{2(\mathcal{P})}\right) d r \\
& -r^{3} \frac{4 \pi}{\tilde{f}_{R}} \int_{0}^{r} e^{\frac{v+\omega}{2}} r^{2}\left(T_{0}^{0(\mathcal{P})}-T_{1}^{1(\mathcal{P})}-2 T_{2}^{2(\mathcal{P})}\right) d r
\end{aligned}
$$

The comparison of Eq.(35) and Eq.(54) provides

$$
\int_{r}^{r \Omega} \frac{e^{\frac{\beta+\alpha}{2}}}{r} Y_{T F} d r=\int_{r}^{r_{\Omega}} \frac{e^{\frac{\beta+\alpha}{2}}}{r}\left[\frac{4 \pi}{\tilde{f}_{R}}\left(P_{r}-P_{\perp}\right)+\mathcal{E}\right] d r
$$

which implies that $Y_{T F}$ is correlated with the fluid distribution's influence of irregular energy density, $f(R)$ corrections, and anisotropic pressure.

\section{The Complexity Factor}

Due to some physical quantities, i.e., inhomogeneity of mass distribution of spherical structure, heat radiations and fluid's viscosity, etc, a CF of a corresponding system can be generated. The simplest system with null CF can be considered normally as a system coupled with an isotropic pressure 
and regular energy density. The origins of $\mathrm{CF}$ here could be anisotropic pressure, MTG terms of Palatini $f(R)$ gravity along with irregular density in the relativistic matter content. One of the scalars, $Y_{T F}$ appearing in Eq. (53) can be called as CF in our case as it appears in the expression of Tolman mass. Our system of partial differential equations includes unknown structural variables. In order to treat such scenario, we restrict our system to enter in the less-complex state. This could be possible by using $Y_{T F}=0$ in the analysis. Under this background, we have

$$
\Pi=\frac{\tilde{f}_{R}}{2 r^{3}} \int_{0}^{r} r^{3}\left(\frac{\rho}{f_{R}}\right)^{\prime} d r+\frac{\tilde{f}_{R}}{2 r^{3}} \int_{0}^{r} r^{3}\left(\frac{T_{0}^{0(\mathcal{P})}}{f_{R}}\right)^{\prime} d r+\frac{1}{2}\left(T_{1}^{1(\mathcal{P})}-T_{2}^{2(\mathcal{P})}\right) .
$$

In this way, we have evaluated the value of $\Pi$. Equation (56) describes the modeling of anisotropic spherical system evolving with zero CF with the extra degrees of freedom mediated by Palatini $f(R)$ terms. To find rest of unknown, it could be helpful to consider viable analytical models from the literature. We shall take a model known widely as Gokhroo-Mehra ansatz (GMA).

Abedi et al. [62 explored the energy-momentum complex for the flat Friedmann-Robertson-Walker manifold within an environment of GR and one of the modified gravity models. It is worthy to stress that our calculated $\mathrm{CF}$ could be related to the energy-momentum complex because it depends on the stress-energy momentum tensor. This suggests that our approach could be related to the definition of gravitational stress-energy pseudo-tensor [63].

\section{The Gokhroo-Mehra Ansatz}

The interior solutions for the spherically symmetric anisotropic fluid configurations having irregular distribution of energy density were studied by Gokhroo and Mehra [64]. They assumed the following choice of energy density as

$$
\rho=\rho_{0}\left(1-\frac{K r^{2}}{r_{\Omega}^{2}}\right),
$$

in which they used $\rho_{0}$ as a constant quantity, along with $K \in(0,1)$. By making use of such selection of energy density, Eq.(19) may be written alternatively as

$$
m(r)=\frac{4 \pi}{\tilde{f}_{R}} \int_{0}^{r} r^{2} T_{0}^{0(\mathcal{P})} d \hat{r}+\frac{4 \pi r^{3}}{3 \tilde{f}_{R}} \rho_{0}\left(1-\frac{3 K r^{2}}{5 r_{\Omega}^{2}}\right)
$$


which upon using Eq.(18) provides

$$
e^{-v}=1+\frac{3 K \xi_{0} r^{4}}{5 r_{\Omega}^{2} \tilde{f}_{R}}-\frac{\xi_{0} r^{2}}{\tilde{f}_{R}}-\frac{8 \pi}{r \tilde{f_{R}}} \int_{0}^{r} r^{2} T_{0}^{0(\mathcal{P})} d r
$$

where $\xi_{0}=8 \pi \rho_{0} / 3$. From Eqs.(14) and (15), we have

$$
\frac{8 \pi}{f_{R}}\left(P_{r}-P_{\perp}+T_{1}^{1(\mathcal{P})}-T_{2}^{2(\mathcal{P})}\right)=e^{-v}\left[\frac{\omega^{\prime}}{2 r}+\frac{1}{r^{2}}+\frac{v^{\prime} \omega^{\prime}}{4}+\frac{v^{\prime}}{2 r}-\frac{\omega^{\prime \prime}}{2}-\frac{\omega^{\prime 2}}{4}\right]-\frac{1}{r^{2}} .
$$

It could be fine to use couple of effective form of new variables as follows

$$
e^{\omega(r)}=\frac{1}{e^{\int\left(\frac{2}{r}-2 z(r)\right) d r}}, \quad e^{v}=\frac{1}{y(r)} .
$$

After using above variables, Eq. (60) turns out to be

$$
y^{\prime}+\left[2 z+\frac{2 z^{\prime}}{z}+\frac{4}{r^{2} z}-\frac{6}{r}\right] y=-\left[\frac{1}{r^{2}}+\frac{8 \pi}{f_{R}}\left(\Pi+T_{1}^{1(\mathcal{P})}-T_{2}^{2(\mathcal{P})}\right)\right] \frac{2}{z} .
$$

The spacetime with its metric coefficients through $z$ and $\Pi$ can be written as 65

$$
\begin{aligned}
d s^{2} & =e^{\int\left(2 z(r)-\frac{2}{r}\right) d r} d t^{2}-r^{2} d \theta^{2}-r^{2} \sin ^{2} \theta d \phi^{2} \\
& +\frac{z^{2}(r) e^{\int\left(2 z(r)-\frac{4}{r^{2} z(r)}\right) d r}}{2 r^{6} \int \frac{e^{\int\left(2 z(r)-\frac{4}{r^{2} z(r)}\right) d r} z(r)\left(\frac{1}{r^{2}}+8 \pi f_{R}^{-1}\left(\Pi+T_{1}^{1(\mathcal{P})}-T_{2}^{2(\mathcal{P})}\right)\right)}{r^{6}} d r+C} d r^{2},
\end{aligned}
$$

where $C$ is an integration constant. In this framework, the values of structural variables are

$$
\begin{aligned}
\frac{4 \pi}{f_{R}}\left(\rho+T_{0}^{0(\mathcal{P})}\right) & =\frac{m^{\prime}}{r} \\
\frac{4 \pi}{f_{R}}\left(P_{r}+T_{1}^{1(\mathcal{P})}\right) & =\frac{\frac{m}{r}-z(2 m-r)-1}{4 \pi r^{2}} \\
\frac{8 \pi}{f_{R}}\left(P_{\perp}+T_{2}^{2(\mathcal{P})}\right) & =z\left(\frac{m}{r^{2}}-\frac{m^{\prime}}{r}\right)+\left(z^{2}+z^{\prime}+\frac{1}{r^{2}}-\frac{z}{r}\right)\left(1-\frac{2 m}{r}\right)
\end{aligned}
$$

These equations have formulated the matter variables in terms of $m, z$ and $f(R)$ dark source terms. After selecting some suitable initial condition, one can solve (analytically or numerically) them for different choices of $z$ and $m$. Then the resultant variables will be expressed in terms of Palatini $f(R)$ dark source terms. Such equations objectively analyze the existence of celestial bodies for $Y_{T F}=0$. 


\section{Concluding Remarks}

In this paper, we study the dynamics of a non-rotating spherically symmetric spacetime which is coupled with a locally anisotropic fluid distribution in a non-linear $f(R)$ theory of gravity. Such a mathematical model can be called intuitively as complex one, thereby indicating a need to explore the corresponding complexity factor. The purpose of this work is to explore this factor in the context of $f(R)$ gravity.

- It is well-known that the static relativistic pressure isotropic spheres with a regular energy density distribution could be regarded as the less complex systems. Therefore, it would be justified to consider null contribution of $\mathrm{CF}$ for those systems. It has been analyzed in GR that one of the structure scalars $Y_{T F}$ is the CF. The same result is found for Palatini $f(R)$ gravity with the difference that the dark source terms of Palatini $f(R)$ terms appearing in the definition of $Y_{T F}$ are slowing down such transition due to their non-attractive nature.

- Another very important result stems form the expression of $Y_{T F}$ is that it contains spherical structural effects coming from the irregular energy density, Palatini $f(R)$ terms and effective form of local anisotropic pressure fabricated in a particular way. This factor has been found to be zero for those systems who evolves with a homogeneous perfect fluid in GR. But in our case the extra curvature Palatini $f(R)$ terms are providing resistance to the system in leaving their homogeneous state. We expect to see such analysis in the presence of electromagnetic field.

- The structure variable $Y_{T F}$ is specifying the role of irregular energy density, Palatini $f(R)$ terms and local anisotropic terms in a particular order.

- The same modified scalar variable has been found to be involved in estimating the digression of the Tolman mass $m_{T}$ for regular spheres, mediated by irregularity in the energy density of the anisotropic matter configurations.

- All of our results reduce to GR [50] under the condition $f(R)=R$.

We have assumed a Palatini $f(R)$ gravity, one can induce further degrees of freedom in the analysis of relativistic ideal and non-ideal configurations. 
Capozziello et al. [66] associated Gauss-Bonnet curvature terms with the stress energy tensor of an ideal matter content. They have also studied the geometric interpretation of the dark components of the cosmological Hubble flow. As an extension in the gravitational component of GR action, the further degrees of freedom in this part can be modeled theoretically as ideal matter configurations sourcing the corresponding equations of motion [67].

\section{Acknowledgments}

This work has been supported financially by National Research Project for Universities (NRPU), Higher Education Commission, Pakistan under research project No. 8754/Punjab/NRPU/R\&D/HEC/2017.

\section{Appendix}

$$
\begin{aligned}
T_{0}^{0(\mathcal{P})} & =\frac{1}{2} f_{\mathcal{P}} e^{\beta}+\frac{e^{\beta-2 \alpha}}{r^{2}}\left[\left(3 \beta^{\prime} \alpha^{\prime}-\beta^{\prime 2}-2 \beta^{\prime \prime}+e^{\alpha}\left(\beta^{\prime 2}+2 \beta^{\prime \prime}-\beta^{\prime} \alpha^{\prime}\right)\right) f_{\mathcal{P}}\right. \\
& \left.+2\left(e^{\alpha}-3\right) \alpha^{\prime} \mathcal{P}^{\prime} f_{\mathcal{P} \mathcal{P}}+4 \mathcal{P}^{\prime \prime}\left(1-e^{\alpha}\right) f_{\mathcal{P} \mathcal{P}}+4 \mathcal{P}^{\prime 2}\left(1-e^{\alpha}\right) f_{\mathcal{P} \mathcal{P} \mathcal{P}}\right] \\
T_{1}^{1(\mathcal{P})} & =-\frac{1}{2} f_{\mathcal{P}} e^{\alpha}+\frac{1}{r^{2}}\left[\left(\beta^{\prime} \alpha^{\prime}\left(1-\frac{3}{e^{\alpha}}\right)+2 \beta^{\prime \prime}\left(\frac{1}{e^{\alpha}}-1\right)+\beta^{\prime 2}\left(\frac{1}{e^{\alpha}}-1\right)\right) f_{\mathcal{P}}\right. \\
& \left.+\left(2 \beta^{\prime} \mathcal{P}^{\prime}\left(1-\frac{3}{e^{\alpha}}\right)\right) f_{\mathcal{P} \mathcal{P}}\right], \\
T_{2}^{2(\mathcal{P})} & =-\frac{1}{2} r^{2} f_{\mathcal{P}}+\left(\frac{\beta^{\prime 2}}{e^{2 \alpha}}-\frac{\beta^{\prime 2}}{e^{\alpha}}-2 \frac{\beta^{\prime \prime}}{e^{\alpha}}+2 \frac{\beta^{\prime \prime}}{e^{2 \alpha}}+\frac{\beta^{\prime} \alpha^{\prime}}{e^{\alpha}}-3 \frac{\beta^{\prime} \alpha^{\prime}}{e^{2 \alpha}}\right) f_{\mathcal{P}} \\
& -\left(\left(r \beta^{\prime 2}+2 r \beta^{\prime \prime}-3 r \beta^{\prime} \alpha^{\prime}\right) \mathcal{P}^{\prime}+2 r \beta^{\prime} \mathcal{P}^{\prime \prime}\right) \frac{f_{\mathcal{P} \mathcal{P}}}{e^{2 \alpha}}-\frac{2 r \beta^{\prime} \mathcal{P}^{\prime 2}}{e^{2 \alpha}} f_{\mathcal{P} \mathcal{P} \mathcal{P}}
\end{aligned}
$$

\section{References}

[1] D. Pietrobon, A. Balbi, and D. Marinucci Phys. Rev. D, vol. 74, p. 043524, 2006. 
[2] T. Giannantonio et al. Phys. Rev. D, vol. 74, p. 063520, 2006.

[3] A. G. Riess et al. Astrophys. J., vol. 659, p. 98, 2007.

[4] J. P. Ostriker and P. J. Peebles Astrophys. J., vol. 186, p. 467, 1973.

[5] A. Refregier Annu. Rev. Astron. Astrophys., vol. 41, p. 645, 2003.

[6] B. P. Abbott et al. Phys. Rev. Lett., vol. 119, p. 161101, 2017.

[7] B. P. Abbott et al. Phys. Rev. Lett., vol. 116, p. 061102, 2016.

[8] B. P. Abbott et al. Phys. Rev. Lett., vol. 119, p. 141101, 2017.

[9] R. Laureijs et al. arXiv preprint arXiv:1110.3193, 2011.

[10] L. Lombriser and N. A. Lima Phys. Lett. B, vol. 765, p. 382, 2017.

[11] T. Baker, E. Bellini, P. G. Ferreira, M. Lagos, J. Noller, and I. Sawicki Phys. Rev. Lett., vol. 119, p. 251301, 2017.

[12] J. M. M. Senovilla and D. Garfinkle Class. Quantum Grav., vol. 32, p. $124008,2015$.

[13] A. Qadir, H. W. Lee, and K. Y. Kim Int. J. Mod. Phys. D, vol. 26, p. 1741001, 2017.

[14] S. Capozziello and V. Faraoni, Beyond Einstein Gravity, vol. 170. Springer Science \& Business Media, 2010.

[15] S. Capozziello and M. De Laurentis Phys. Rep., vol. 509, p. 167, 2011.

[16] K. Bamba, S. Capozziello, S. Nojiri, and S. D. Odintsov Astrophys. Space Sci., vol. 342, p. 155, 2012.

[17] M. J. Khan, G. Shabbir, and M. Ramzan Mod. Phys. Lett. A, vol. 34, p. 1950189, 2019.

[18] S. K. Sahu, S. K. Tripathy, P. K. Sahoo, and A. Nath Chin. J. Phys., 2017.

[19] S. Nojiri and S. D. Odintsov Int. J. Geom. Meth. Mod. Phys., vol. 4, p. 115, 2007. 
[20] E. Elizalde, S. Nojiri, S. D. Odintsov, L. Sebastiani, and S. Zerbini Phys. Rev. D, vol. 83, p. 086006, 2011.

[21] K. Bamba, C.-Q. Geng, and C.-C. Lee J. Cosmol. Astropart. Phys., vol. 2010, p. 021, 2010.

[22] M. J. S. Houndjo, M. E. Rodrigues, N. S. Mazhari, D. Momeni, and R. Myrzakulov Int. J. Mod. Phys. D, vol. 26, p. 1750024, 2017.

[23] Z. Yousaf, M. Sharif, M. Ilyas, and M. Z. Bhatti Int. J. Geom. Meth. Mod. Phys., vol. 15, p. 1850146, 2018.

[24] S. Nojiri and S. D. Odintsov Phys. Lett. B, vol. 631, p. 1, 2005.

[25] M. Z. Bhatti, M. Sharif, Z. Yousaf, and M. Ilyas Int. J. Mod. Phys. D, vol. 27, p. 1850044, 2018.

[26] Z. Yousaf Astrophys. Space Sci., vol. 363, p. 226, 2018.

[27] Z. Yousaf Eur. Phys. J. Plus, vol. 134, p. 245, 2019.

[28] M. F. Shamir and M. Ahmad Mod. Phys. Lett. A, vol. 34, p. 1950038, 2019.

[29] K. Bamba, S. Nojiri, and S. D. Odintsov arXiv preprint arXiv:1302.4831, 2013.

[30] Z. Yousaf, K. Bamba, and M. Z. Bhatti Phys. Rev. D, vol. 93, p. 124048, 2016.

[31] Z. Yousaf, K. Bamba, and M. Z. Bhatti Phys. Rev. D, vol. 93, p. 064059, 2016.

[32] S. Nojiri, S. Odintsov, and V. Oikonomou Phys. Rep., vol. 692, p. 1, 2017.

[33] S. Nojiri and S. D. Odintsov arXiv preprint arXiv:0807.0685, 2008.

[34] C. Bambi, A. Cardenas-Avendano, G. J. Olmo, and D. Rubiera-Garcia Phys. Rev. D, vol. 93, p. 064016, 2016.

[35] G. J. Olmo and D. Rubiera-Garcia Phys. Rev. D, vol. 84, p. 124059, 2011. 
[36] G. J. Olmo and D. Rubiera-Garcia Universe, vol. 1, p. 173, 2015.

[37] Z. Yousaf Mod. Phys. Lett. A, vol. 34, p. 1950333, 2019.

[38] R. Penrose, "Singularities and time-asymmetry," in General Relativity: An Einstein Centenary Survey (W. Israel and S. W. Hawking, eds.), p. 581, Cambridge University Press, 1979.

[39] D. M. Eardley and L. Smarr Phys. Rev. D, vol. 19, p. 2239, 1979.

[40] L. Herrera, A. Di Prisco, J. L. Hernández-Pastora, and N. O. Santos Phys. Lett. A, vol. 237, p. 113, 1998.

[41] L. Herrera, A. Di Prisco, J. Martin, J. Ospino, N. O. Santos, and O. Troconis Phys. Rev. D, vol. 69, p. 084026, 2004.

[42] K. Bamba, S. Nojiri, and S. D. Odintsov Phys. Lett. B, vol. 698, p. 451, 2011.

[43] Z. Yousaf, K. Bamba, and M. Z. Bhatti Phys. Rev. D, vol. 95, p. 024024, 2017.

[44] Z. Yousaf, M. Z. Bhatti, and A. Rafaqat Can. J. Phys., vol. 95, p. 1246, 2017.

[45] Z. Yousaf and M. Z. Bhatti Int. J. Geom. Meth. Mod. Phys., vol. 15, p. $1850160,2018$.

[46] Z. Yousaf, M. Z. Bhatti, and R. Saleem Eur. Phys. J. Plus, vol. 134, p. 142, 2019.

[47] M. Z. Bhatti, Z. Yousaf, and M. Yousaf Phys. Dark Universe, vol. 28, p. 100501, 2020.

[48] Z. Yousaf, Phys. Dark Universe, vol. 28, p. 100509, 2020.

[49] Z. Yousaf, M. Z. Bhatti, and H. Asad Phys. Dark Universe, vol. 28, p. 100527, 2020.

[50] L. Herrera Phys. Rev. D, vol. 97, p. 044010, 2018.

[51] G. Abbas and H. Nazar Eur. Phys. J. C, vol. 78, p. 510, 2018. 
[52] L. Herrera, A. Di Prisco, and J. Ospino Phys. Rev. D, vol. 98, p. 104059, 2018.

[53] M. Sharif and A. Majid Chin. J. Phys., vol. 61, p. 38, 2019.

[54] Z. Yousaf, M. Z. Bhatti, T. Naseer and I. Ahmad Phys. Dark Universe, vol. 29, p. 100581, 2020.

[55] Z. Yousaf, M. Z. Bhatti and T. Naseer Eur. Phys. J. Plus, vol. 135, p. 323, 2020.

[56] S. Capozziello, T. Harko, T. S. Koivisto, F. S. N. Lobo, and G. J. Olmo Phys. Rev. D, vol. 86, p. 127504, 2012.

[57] C. W. Misner and D. H. Sharp Phys. Rev., vol. 136, p. B571, 1964.

[58] R. C. Tolman Phys. Rev., vol. 35, p. 875, 1930.

[59] G. Darmois Gauthier-Villars, Paris, vol. 25, 1927.

[60] L. Herrera and N. O. Santos Phys. Rep., vol. 286, p. 53, 1997.

[61] L. Herrera, J. Ospino, A. Di Prisco, E. Fuenmayor, and O. Troconis Phys. Rev. D, vol. 79, p. 064025, 2009.

[62] H. Abedi, A. M. Abbassi, S. Capozziello Ann. Phys-New York, vol. 405, p. 54, 2019.

[63] S. Capozziello, C. Maurizio, and T. Maria Ann. Phys-Berlin, vol. 529, p. $1600376,2017$.

[64] M. K. Gokhroo and A. L. Mehra Gen. Relativ. Gravit., vol. 26, p. 75, 1994.

[65] L. Herrera, J. Ospino, and A. Di Prisco Phys. Rev. D, vol. 77, p. 027502, 2008.

[66] S. Capozziello, C. A. Mantica, and L. G. Molinari Int. J. Geom. Meth. Mod. Phys., vol. 16, p. 1950133, 2019.

[67] S. Capozziello, C. A. Mantica, and L. G. Molinari Int. J. Geom. Meth. Mod. Phys., vol. 16, p. 1950008, 2019. 